\title{
SUR LES APPLICATIONS HARMONIQUES
}

\author{
JEAN-CLAUDE MITTEAU
}

\section{Principaux résultats}

Nous considérons deux variétés riemanniennes $(M, g)$ et $\left(M^{\prime}, g^{\prime}\right)$; nous supposons $M$ compacte et $\left(M^{\prime}, g^{\prime}\right)$ complète. On pose $n=\operatorname{dim}$ M. Variétés, métriques et connexions sont toujours supposées $C^{\infty}$.

Soit $f \in C^{1}\left(M, M^{\prime}\right)$. On associe à $f$ sa densité d'énergie $e(f) \in C^{0}(M)$

$$
e(f)(p)=|T f(p)|^{2},
$$

en coordonnées locales $e(f)=\left(g^{\prime} \circ f\right)_{\alpha \beta} \partial_{i} f^{\alpha} \partial_{j} f^{\beta} g^{i j}$. L'énergie $E(f)$ est l'intégrale

$$
E(f)=\int_{M} e(f)(p) d v(p),
$$

$v$ étant la mesure canonique de volume de la variété $(M, g)$. Un ensemble $K \subset C^{1}\left(M, M^{\prime}\right)$ est dit $C^{1}$-borné si $\operatorname{Im}(K)$ dans $\left(M^{\prime}, g^{\prime}\right)$ et $\{e(f)(p) \mid f \in K, p \in M\}$ sont des ensembles bornés.

Considérons une classe d'homotopie $\mathscr{C}$ d'applications de $M$ dans $M^{\prime}$.

(1.2) Théorème. Une condition nécessaire et suffisante pour qu'il existe un minimum absolu de l'énergie (1.1) dans $\mathscr{C}$ est: on peut trouver $K \subset C^{1}\left(M, M^{\prime}\right)$, $K C^{1}$-borné, tel que

$$
\operatorname{Inf}_{f \in K} E(f)=\operatorname{Inf}_{f \in \mathscr{R} \cap C^{1}\left(M, M^{\prime}\right)} E(f) .
$$

On dit qu'une application $f \in C^{1}\left(M, M^{\prime}\right)$ est harmonique si $f$ est un extremum de l'énergie (1.1). Si $f \in C^{2}\left(M, M^{\prime}\right)$, il est équivalent de dire que $\tau(f)=0$, où $\tau$ est le champ de tensions de l'application $f$ [1, chapitre 1, paragraphe 2A].

(1.3) Théorème. Supposons $\left(M^{\prime}, g^{\prime}\right)$ compacte. Il existe un réel $\varepsilon_{1}>0$ tel que si on peut trouver $f \in \mathscr{C} \cap C^{1}\left(M, M^{\prime}\right)$ vérifiant $e(f) \leq \varepsilon_{1}$, alors $\mathscr{C}$ contient au moins une application harmonique.

Dans le cas où $\left(M^{\prime}, g^{\prime}\right)$ est une variété à courbure sectionnelle non positive, on peut prendre $\varepsilon_{1}=\infty$. Ce théorème est donc une généralisation du deuxième corollaire du théorème 11. A de [1].

(1.4) Théorème. Supposons que $(M, g)$ vérifie la propriété (5.11). Supposons que $\left(M^{\prime}, g^{\prime}\right)$ ait une courbure sectionnelle majorée par $R \geq 0$. Il existe un réel $\varepsilon_{2}>0$ tel que si $f \in C^{2}\left(M, M^{\prime}\right)$ vérifie

Communicated by A. Lichnerowicz, August 12, 1972. 
i) $f$ est harmonique,

ii) $e(f) \leq \varepsilon_{2}$,

alors $f$ est une application constante.

Enfin, les théorèmes 4.9 et 5.10 donnent cetains résultats sur les solutions de l'équation d'évolution naturellement associée au problème.

\section{Méthodes}

A. L'opérateur $\tau$ n'est pas linéaire mais polynômial, au sens de [8, chapitre 16].

Nous remplaçons la recherche des solutions de l'équation $\tau(\mu)=0$ par la recherche des limites, pour $t \rightarrow \infty$, des solutions du problème de Cauchy

$$
\begin{gathered}
\left.\frac{\partial \mu}{\partial t}(p, t)-\tau\left(\mu_{t}\right)(p)=0, \quad \forall(p, t) \in M \times\right] 0, T[ \\
\dot{\mu}_{0}=f \in C^{0}\left(M, M^{\prime}\right) \text { donné } .
\end{gathered}
$$

Les solutions $\mu$ de ce problème sont cherchées dans $C^{0}\left(M \times[0, \alpha), M^{\prime}\right) \cap$ $\left.\left.C^{\infty}(M \times] 0, \alpha\right), M^{\prime}\right) . \mu_{t}$ est l'application partielle $\mu(\cdot, t)$. On sait qu'il y a unicité des solutions [6].

Le théorème (3.1) est un résultat d'existence uniforme des solutions de (2.1), lorsqu'on prend la condition de Cauchy $f$ dans un ensemble $K C^{1}$-borné. Il joue un rôle central dans les démonstrations.

Nous utilisons dans la suite le fait que $E\left(\mu_{t}\right)$ est une fonction décroissante du paramètre, lorsque $\mu$ est solution de (2.1).

B. Notons $\rho$ la distance riemannienne et $\theta$ la distance dans $C^{0}\left(M, M^{\prime}\right)$ :

$$
\theta(f, g)=\operatorname{Sup}_{x \in M} \rho(f(x), g(x)) .
$$

La suite de ce paragraphe utilise largement les notations et résultats de [6]. Si $f \in C^{0}\left(M, M^{\prime}\right)\left(\right.$ resp. $\left.\mu \in C^{0}\left(M \times[0, T], M^{\prime}\right)\right)$, on désigne par $V_{f}$ (resp. $\left.V_{\mu}\right)$ le fibré image réciproque $f^{*} T M^{\prime}$ (resp. $\mu^{*} T M^{\prime}$ ). Ces fibrés sont munis canoniquement de produits scalaires et de connexions linéaires. Il en est de même des fibrés $L^{(k)}\left(T M, V_{f}\right)$ (resp. $L^{(k)}\left(T M \times T[0, T], V_{\mu}\right)$ ). Si $f \in C^{k}\left(M, M^{\prime}\right)$ (resp. $\left.\mu \in C^{k}\left(M \times[0, T], M^{\prime}\right)\right)$, la dérivée d'ordre $k, f^{(k)} \in L^{(k)}\left(T M, V_{f}\right)$ (resp. $\mu^{(k)} \in$ $\left.L^{(k)}\left(T M \times T[0, T], V_{\mu}\right)\right)$.

(2.2) Définition. Un ensemble $K \subset C^{k}\left(M, M^{\prime}\right)$ est dit $C^{k}$-borné si

i) $\operatorname{Im}(K)$ est borné dans $\left(M^{\prime}, g^{\prime}\right)$;

ii) il existe une constante $C$ telle que ${ }^{1}$

$$
\left|\mu^{(j)}(p)\right| \leq C, \quad j=1, \cdots, k, \quad p \in M
$$

\footnotetext{
${ }^{1}$ nous donnerons éventuellement le même nom, dans la suite, à des constantes de valeurs différentes.
} 
On définit, sur les section $C^{2}\left(L^{(k)}\left(T M \times T[0, T], V_{\mu}\right)\right)$, l'opérateur

$$
\Lambda=\nabla_{t}-\nabla_{i} \nabla^{i}
$$

les indices $i=1, \cdots, n$ désignant les coordonnées dans $M$, dites aussi spatiales, et $t$ la coordonnées dans [0,T], dite aussi temporelle. Si $\mu$ est solution de (2.1), $\mu^{(k)}$ vérifie une équation

$$
\Lambda \mu^{(k)}=\psi_{k}
$$

$\psi_{k}$ étant borné si d'une part les tenseurs de courbure de $(M, g)$ et $\left(M^{\prime}, g^{\prime}\right)$ et leurs dérivées sont bornés et si d'autre part, $\mu$ est $C^{k}$-borné et $\partial \mu / \partial t$ est $C^{k-2}$ borné [6].

C. Pour inverser $\Lambda$, on utilisera les opérateurs pseudo-différentiels définis par le noyau de Green $H$ associé à $A$. $H$ est un bitenseur, dans le sens de [4], paragraphe 3 . Nous noterons $*$ et $\cdot$ les convolutions suivantes

$$
\begin{aligned}
& \left(H_{t_{0}}^{t} * X\right)(p, t)=\int_{t_{0}}^{t} \int_{M} H(p, t ; q, \tau)[X(q, \tau)] d v(q) d \tau, \\
& \left(H_{t_{0}}^{t} \cdot X\right)(p, t)=\int_{M} H\left(p, t ; q, t_{0}\right)\left[X\left(q, t_{0}\right)\right] d v(q) .
\end{aligned}
$$

Le noyau $H$ vérifie les propriétés usuelles de majoration. On notera $H^{(i)}$ les itérées de $H$ :

$$
\begin{aligned}
H^{(i)}\left(p, t_{1} ; q, t_{0}\right) & =\int_{t_{0}}^{t_{1}} \int_{M} H\left(p, t_{1} ; m, \tau\right) \circ H^{(i-1)}\left(m, \tau ; q, t_{0}\right) d v(m) d \tau, \\
H^{(1)} & =H .
\end{aligned}
$$

Pour l'étude et les propriétés de $H$, nous renvoyons à [9] et [6], chapitre 3 et annexes 1 et 2 .

D. Pour pouvoir appliquer dans la résolution de (2.1) le noyau $H \mathrm{du}$ paragraphe précédent, nous représentons localement au moyen de l'application exponentielle la variété $C^{k}\left(M, M^{\prime}\right)$ par les sections $C^{k}$ d'une fibré $V_{g}$, $g \in C^{\infty}\left(M, M^{\prime}\right)$, [2]. Notons $\mathscr{V}_{g, \rho} \subset C^{0}\left(V_{g}\right)$ la boule de rayon $\rho$. Pour $\rho$ assez petit, $\operatorname{Exp}_{g}: \mathscr{V}_{g, \rho} \rightarrow C^{0}\left(M, M^{\prime}\right)$ est un homéomorphisme sur un ouvert de $C^{0}\left(M, M^{\prime}\right)$, [2].

Nous avons obtenu dans [6] le résultat suivant: il existe un réel $\rho_{0}>0$, ne dépendant que de l'ensemble $\operatorname{Im}(g) \subset M^{\prime}$, tel que l'application $\operatorname{Exp}_{g}: \mathscr{V}_{g, \rho_{0}}$ $\cap C^{2}\left(V_{g}\right) \rightarrow C^{2}\left(M, M^{\prime}\right)$ se prolonge en un isomorphisme des opérateurs différentiels (non-linéaires) d'ordre 2.

Ainsi, pour $\theta(\mu, g) \leq \rho_{0}$, le problème (2.1) est équivalent par $\mu=\operatorname{Exp}_{g} X$ à

$$
\begin{gathered}
\Lambda X=\Phi(X, \nabla X), \quad X \in\left(C^{2}, C^{1}\right)\left(V_{g} \times\left[0, T[), \quad\|X\|_{L^{\infty}} \leq \rho_{0},\right.\right. \\
X_{0}=F \in \mathscr{V}_{g, \rho_{0}} \text { donné },
\end{gathered}
$$


[6, paragraphe (2.8)]. $\nabla X$ est la dérivée covariante spatiale de $X$ dans la connexion naturelle sur $V_{g}$.

La méthode permet d'obtenir en outre des majorations. Celles dont nous nous servirons sont

$$
\begin{gathered}
\|\Phi(X, \nabla X)+\tau(g)\|_{L^{\infty}\left(V_{g}\right)} \leq C\|X\|_{L^{\infty}\left(V_{g}\right)}\left[1+\|\nabla X\|_{L^{\infty}\left(L\left(T M, V_{g}\right)\right.}^{2}\right] \\
\|\nabla X\|_{L^{\infty}\left(L\left(T M, V_{g}\right)\right)}^{2} \leq C\left[1+\left\|e\left(\operatorname{Exp}_{g} X\right)\right\|_{L^{\infty}(M)}\right] \\
\left\|e\left(\operatorname{Exp}_{g} X\right)\right\|_{L^{\infty}(M)} \leq C\left[1+\|\nabla X\|_{L^{\infty}\left(L\left(T M, V_{g}\right)\right)}^{2}\right]
\end{gathered}
$$

à condition que $\operatorname{Exp}_{g} X$ soit dans $\mathscr{V}_{g, \rho_{0}}$.

\section{Existence uniforme des solutions de l'équation d'évolution}

Soient $K \subset C^{0}\left(M, M^{\prime}\right)$ et $\alpha>0$. Définissons

$$
\begin{gathered}
\left.\mathscr{S}_{\alpha}(K)=\left\{\mu \in C^{0}\left(M \times[0, \alpha], M^{\prime}\right) \cap C^{\infty}(M \times] 0, \alpha\right], M^{\prime}\right) \mid \mu \\
\text { est solution de } \left.(2.1), \mu_{0} \in K\right\} .
\end{gathered}
$$

Cet ensemble est éventuellement vide. On note $\omega_{\alpha}: \mathscr{S}_{\alpha}(K) \rightarrow C^{0}\left(M, M^{\prime}\right) 1^{\prime}$ application définie par $\omega_{\alpha}(\mu)=\mu_{0}$.

(3.1) Théorème. Supposons $K \subset C^{1}\left(M, M^{\prime}\right)$ et $C^{1}$-borné. On peut trouver $\alpha>0$ tel que $\omega_{\alpha}$ soit surjective. On a, en outre, les propriétés suivantes

a) $\left\{\mu_{t} \mid \mu \in \mathscr{S}_{\alpha}(K), t \in[0, \alpha]\right\}$ est $C^{1}$-borné ;

b) pour tout $\varepsilon>0$, on peut trouver $\eta, 0<\eta<\alpha$, tel que

$$
\theta\left(\mu_{t}, \mu_{0}\right) \leq \varepsilon, \quad \forall \mu \in \varphi_{\alpha}(K), \quad \forall t \in[0, \eta] ;
$$

c) fixons un entier $k \geq 1$ et un réel $\alpha^{\prime}, \quad 0<\alpha^{\prime} \leq \alpha$; l'ensemble $\left\{\mu_{t} \mid \mu \in \mathscr{S}_{\alpha}(K), \alpha^{\prime} \leq t \leq \alpha\right\}$ est $C^{k}$-borné.

(3.2) Remarque. Ce théorème est encore vérifié si on remplace la condition " $\left(M^{\prime}, g^{\prime}\right)$ est complète" par la condition plus faible " $K$ étant fixé, il existe un réel $\delta>0$, tel que l'ensemble

$$
K_{\delta}=\left\{x \in M^{\prime} \mid \exists y \in \operatorname{Im}(K), \rho(x, y)<\delta\right\}
$$

soit relativement compact".

(3.4) Remarque. Une conséquence du théorème (3.1) et de la formule (3.6) est que tout ensemble $C^{1}$-borné d'applications de $M$ dans $M^{\prime}$ peut être uniformément $C^{1}$-approché par un ensemble $C^{k}$-borné [7].

Démonstration du théorème (en quatre étapes). $1 . K \subset C^{0}\left(M, M^{\prime}\right)$ est relativement compact. Première conséquence, l'ensemble $K_{\delta}$, défini en (3.3), est relativement compact dans $\left(M^{\prime}, g^{\prime}\right)$; le tenseur de courbure de cette variété et toutes ses dérivées seront donc bornés. Deuxième conséquence, il existe un 
réel $\rho_{0}>0$ fixe tel que pour toute application $g \in C^{\infty}\left(M, M^{\prime}\right)$ telle que $\operatorname{Im}(g)$ $\subset K_{\delta}$, on puisse appliquer les résultats de paragraphe 2, D. Prenons $\rho_{1}=$ $\frac{1}{2} \rho_{0} / C_{1}, C_{1}$ étant un majorant sur $[0, T]$ de la norme de l'opérateur

$$
X \mapsto H_{0}^{t} \cdot X, \quad X \in C^{0}\left(V_{g}\right) .
$$

On sait que ce majorant existe et ne dépend pas de $g$. Il existe un nombre fini de boules $\mathscr{V}_{g_{i}, \rho_{1}}$ telles que $K \subset \cup_{i} \mathscr{V}_{g_{i}, \rho_{1}}$. On peut donc supposer pour simplifier que $K$ est inclus dans un seul voisinage tubulaire. De (2.6) on déduit que si $\operatorname{Exp}_{g} F \in K$, alors on peut trouver un réel $\xi_{0}$ tel que $\|\nabla F\|_{L^{\infty}\left(V_{g}\right)}<\xi_{0}$.

2. Substituons au problème (2.1) le problème (2.4). Soit $\alpha, 0<\alpha<T$. On considère le convexe fermé borné $\mathscr{A}_{\xi, \alpha}$ de $C^{1}\left(V_{g}\right)$ défini par

$$
\left\|X_{t}\right\|_{L^{\infty}(V g)} \leq \rho_{0}, \quad\left\|\nabla X_{t}\right\|_{L^{\infty}(L(T M, V g))} \leq \xi, \quad \forall t \in[0, \alpha]
$$

Appliquons dans $V_{g}$ la formule de Green associée à l'opérateur $\Lambda$; on définit une application $\Psi: \mathscr{A}_{\xi, \alpha} \rightarrow C^{1}\left(V_{g} \times[0, \alpha]\right)$ par $\Psi(X)=Y$ avec

$$
Y_{t}=H_{0}^{t} * \Phi(X, \nabla X)+H_{0}^{t} \cdot X_{0} .
$$

Si $\hat{X}$ est point fixe de $\Psi$, et si $\hat{X}$ est, pour $t>0,\left(C^{2}, C^{1}\right)$, alors $\hat{X}$ est solution de (2.4) [9].

$Y_{t}$ est de classe $C^{1}$ (spatiale). En effet on peut dériver les convolutions. On obtient

$$
\nabla Y_{t}=\nabla H_{0}^{t} * \Phi(X, \nabla X)+\nabla H_{0}^{t} \cdot X_{0}
$$

Le bitenseur $H$ est dérivé par rapport à sa première variable. D'après le lemme (3.9), $\mathscr{U}$ étant un bitenseur $C^{\infty}$ quelconque, ceci se met sous la forme

$$
\nabla_{z} Y_{t}=\nabla_{z} H_{0}^{t} * \Phi(X, \nabla X)+L_{t} \cdot \nabla_{\mathscr{L} Z} X_{0}+L_{t}^{\prime} \cdot X_{0}, \quad Z \in T_{p} M .
$$

Pour tout $\varepsilon^{\prime}>0$, on peut trouver une valeur de $\alpha$ telle que (2.5), (3.5) et (3.6) fournissent, pour $t \in[0, \alpha]$, les majorations

$$
\begin{aligned}
& \|Y\|_{L^{\infty}} \leq \varepsilon^{\prime}\left(1+\xi^{2}\right)+C_{1} \rho_{1}, \\
& \|\nabla Y\|_{L^{\infty}} \leq \varepsilon^{\prime}\left(1+\xi^{2}\right)+C_{2}\left(\xi_{0}+\rho_{1}\right) .
\end{aligned}
$$

$\rho_{1}$ et $\xi_{0}$ sont donnés. Prenons $\xi=2 C_{2}\left(\xi_{0}+\rho_{1}\right)$. Si $\varepsilon^{\prime}$ est choisi assez petit, on aura certainement

$$
\varepsilon^{\prime}\left(1+\xi^{2}\right)+C_{1} \rho_{1} \leq \rho_{0}, \quad \varepsilon^{\prime}\left(1+\xi^{2}\right)+C_{2}\left(\xi_{0}+\rho_{1}\right) \leq \xi .
$$

Il existe donc des valeurs de $\xi$ et $\alpha$ telles que $\Psi$ soit une application de $\mathscr{A}_{\xi, \alpha}$ dans lui-même. Remarquons même que l'on peut déduire des formules (50) et (52) de [6] que $\Phi$ est lipschitzien; par conséquent, on peut trouver une valeur de $\alpha$ telle que $\Psi$ soit une contraction, ce qui redémontre l'unicité. 
3. Soit $\hat{X}$ le point fixe de $\Psi$. La dérivée spatiale $\nabla \hat{X}$ est bornée. Si $\hat{X}$ vérifie une condition de Hölder, il en est de même de $\Phi(\hat{X}, \nabla \hat{X})$. Par le raisonnement habituel, on en déduit que

a) $\hat{X}$ vérifie une condition de Hölder. Ceci donne la propriété b) du théorème et

ß) $\hat{X}$ est de classe $\left(C^{2}, C^{1}\right)$ et donc solution de (2.4).

Par construction du convexe $\mathscr{A}_{\xi, \alpha}$, on voit que $\mu=\operatorname{Exp}_{g} \hat{X}$ est défini ; c'est donc une solution de (2.1). De la formule (2.6), on déduit le a) du théorème.

4. On démontre par la même méthode, appliquée aux prolongements de l'équation de (2.1) que, pour $t>0, \mu$ est $C^{\infty}$. Fixons un entier $k \geq 1$. L'ensemble $\left\{\mu_{t} \mid \mu \in \mathscr{S}_{\alpha}(K), 0 \leq t \leq \alpha\right\}$ est $C^{1}$-borné. Raisonnons par récurrence. Posons $\alpha_{p}=p \alpha^{\prime} / k$. Supposons l'ensemble $\left\{\mu_{t} \mid \mu \in \mathscr{S}_{\alpha}(K), \alpha_{p-1} \leq t \leq \alpha\right\} \mathrm{C}^{p-1}$ borné et vérifiant une condition de Hölder uniforme. On a, pour $t>\alpha_{p-1}$

$$
\mu_{t}^{(p)}=\nabla \mu_{t}^{(p-1)}=\nabla H_{\alpha_{p-1}}^{t} * \psi_{p-1}+\nabla H_{\alpha_{p-1}}^{t} \cdot \mu_{\alpha_{p-1}}^{(p-1)} .
$$

On en déduit que $\mu^{(p)}$ est borné et vérifie une condition de Hölder uniforme, pour $t \in\left[\alpha_{p}, \alpha\right]$ et tout $\mu$ dans $\mathscr{S}_{\alpha}(K),[10]$.

(3.7) Remarque. Si on a $K \subset C^{k}\left(M, M^{\prime}\right), C^{k}$-borné. les conclusions a), pour $\mu^{(i)}, 1 \leq i \leq k$, et $\mathrm{b}$ ), pour $\mu^{(i)}, i>k$ restent vérifiées.

Soit $\left\{f_{i} \mid i \in N\right\}$ une suite $C^{1}$-bornée. Par le théorème (3.1), on sait que, pour tout $i$, le problème de Cauchy (2.1) admet une solution $\mu^{i}$ dans $\mathscr{S}_{\alpha}\left(\left\{f_{i} \mid i \in N\right\}\right)$. A chaque $f_{i}$ associons $g_{i} \in C^{\infty}\left(M, M^{\prime}\right)$ par $g_{i}=\mu^{i}(\cdot, \alpha)$.

Corollaire. Pour tout entier $k \geq 1$, il existe une sous-suite $j: N \rightarrow N$ telle que la suite $g_{j(i)} C^{k}$-converge, pour $i \rightarrow \infty$, vers une application $g_{\infty}$. En outre

$$
E\left(g_{\infty}\right) \leq \liminf _{i \rightarrow \infty} E\left(f_{i}\right)
$$

Démonstration. On extrait une première sous-suite $j_{1}$ telle que $\lim _{i \rightarrow \infty} f_{j_{1}(i)}$ $=\lim \inf _{i \rightarrow \infty} E\left(f_{i}\right)$. Cette sous-suite est encore $C^{1}$-bornée. Appliquons le théorème $(3.1), \mathrm{c})$. La suite $g_{j_{1}(i)}$ est $C^{k+1}$-bornée. On prend une sous-suite qui $C^{k}$-converge. On sait d'autre part que $E\left(g_{i}\right) \leq E\left(f_{i}\right)$. Quitte à prendre une nouvelle sous-suite, l'inégalité passe à la limite. q.e.d.

De ce corollaire, on déduit le théorème (1.2). Notons $E(\mathscr{C})=$ $\operatorname{Inf}_{f \in \mathscr{E} \cap C^{1}\left(M, M^{\prime}\right)} E(f)$.

Démonstration de théorème (1.2). pour tout $i \in N$, on peut trouver $f_{i}$ tel que $E(\mathscr{C}) \leq E\left(f_{i}\right) \leq E(\mathscr{C})+1 / i$. Le résultat est une conséquence de la remarque (3.7). q.e.d.

La situation des exemples des paragraphes $4, E$ et $10, D$ de [1] est donc générale [7].

Nous avons utilisé dans la démonstration du théorème (3.1) un lemme auxiliaire dont nous donnons maintenant la démonstration. Celle-ci utilise la construction explicite du noyau $H$, faite dans [6]. Nous renvoyons donc le lecteur à ce travail. 
Soit $\mathscr{U} \in C^{\infty}\left(L\left(V_{g} \times[0, \alpha], V_{g} \times[0, \alpha]\right)\right)$, un bitenseur.

(3.9) Lemme. Il existe deux noyaux $L_{t}$ et $L_{t}^{\prime}$ tels que, pour tout $X \in C^{1}\left(V_{g}\right)$ et $t \in] 0, \alpha]$, on ait

i) $\nabla_{Z} H_{0}^{t} \cdot X=L_{t} \cdot \nabla_{q Z} X+L_{t}^{\prime} \cdot X, \quad Z \in T_{p} M$,

ii) pour $|Z|<1$, les opérateurs sur $C^{0}\left(V_{g}\right)$ définis par

$$
X \mapsto L_{t} \cdot X, \quad X \mapsto L_{t}^{\prime} \cdot X
$$

sont bornés, uniformément en $t \in] 0, \alpha]$.

Remarquons que la norme de ces opérateurs dépend de $(M, g)$ et de la connexion dans $V_{g}$ et qu'évidemment, il n'y a pas unicité de cette décomposition.

Démonstration. Une façon de construire $H$ est de considérer le noyau $K=E \mathscr{T}$ où $E$ est le noyau de Gauss et $\mathscr{T}$ un bitenseur de transport $C^{\infty}$. $H$ s'écrit $H=K+K * \mathrm{Q}, \mathrm{Q}$ étant majoré par

$$
\left.|\mathrm{Q}(p, t ; q, u)| \leq C_{\alpha}(t-u)^{-\alpha}[\rho(p \cdot q)]^{2 \alpha-n}, \quad \alpha \in\right] 0,1[
$$

quelconque. On a

$$
\nabla_{Z} H=\nabla_{Z} K+\nabla_{Z} K * \mathrm{Q} .
$$

Des majorations usuelles sur $E$ [9], on déduit que l'opérateur défini par

$$
X \mapsto\left(\nabla_{Z} K * \mathrm{Q}\right) \cdot X
$$

est uniformément borné.

Introduisons la dérivée d'un bitenseur par rapport à la deuxième variable; notons $\nabla^{\prime}$ cette dérivation. Il vient

$$
\nabla_{Z} K \cdot X=\left(\nabla_{Z} K-\nabla_{\mathscr{} Z}^{\prime} K\right) \cdot X+\nabla_{{ }^{\prime} Z}^{\prime} K \cdot X
$$

Or $\nabla_{Z} K-\nabla_{\mathscr{H} Z}^{\prime} K=\left(\nabla_{Z} E-\nabla_{\mathscr{H} Z}^{\prime} E\right) \mathscr{T}+E\left(\nabla_{Z} \mathscr{T}-\nabla_{\mathscr{H} Z}^{\prime} \mathscr{T}\right) . \mathscr{T}$ étant de classe $C^{\infty}$, ainsi que $\mathscr{U}$, la courbure de $V_{g}$ étant bornée, l'opérateur

$$
X \mapsto\left[E\left(\nabla_{Z} \mathscr{T}-\nabla_{\mathscr{L} Z}^{\prime} \mathscr{T}\right)\right]_{0}^{t} \cdot X
$$

est borné uniformément en $t$, car il en est ainsi de l'opérateur

$$
\varphi \mapsto E_{0}^{t} \cdot \varphi, \quad \varphi \in C^{0}(M) .
$$

Il résulte des calculs explicites effectués dans [6], chapitre 2, que

$$
\left(\nabla_{Z} E-\nabla_{q_{Z}}^{\prime} E\right)(p, t ; q, t-u) \leq C u^{-1}[\rho(p, q)]^{2} E(p, t ; q, t-u) .
$$

L'opérateur

$$
X \mapsto\left[\left(\nabla_{Z} E-\nabla_{\mathscr{L} Z}^{\prime} E\right) \mathscr{T}\right]_{0}^{t} \cdot X
$$

est donc lui aussi uniformément borné. 
Enfin, on peut écrire

$$
\nabla_{{ }^{\prime} Z}^{\prime} K \cdot X=\int_{M} \nabla_{{ }^{\prime} Z}^{\prime}\{K(p, t ; q, 0)[X(q, 0)]\} d v(q)+K \cdot \nabla_{\mathscr{Q} Z} X
$$

On pose $L_{t}=K_{0}^{t}$. Il est borné pour la même raison que (3.11). Il reste à évaluer l'intégrale. Introduisons le pseudo-groupe à un paramètre $u$ engendré par $\mathscr{U} Z$, et noté $\mathrm{D}$. Posons $Y: M \rightarrow T_{p} M$ défini par

$$
Y(q)=K(p, t ; q, 0)[X(q, 0)] .
$$

On a $\nabla_{\mathscr{}} Y=(\partial / \partial u)[Y \circ D(u)]_{u=0}$. Effectuons le changement de variables $q \mapsto D(u)(q)=q^{\prime}$. Il vient

$$
\int_{M} \nabla_{\mathscr{U} Z} Y=\frac{d}{d u} \int_{M} Y\left(q^{\prime}\right)\left|T D^{-1}(u)\right|\left(q^{\prime}\right) d v\left(q^{\prime}\right),
$$

et donc

$$
\int_{M} \nabla_{\mathscr{L} Z} Y=\int_{M} Y\left(q^{\prime}\right)\left[\frac{\partial}{\partial u}\left|T D^{-1}(u)\right|\right]\left(q^{\prime}\right) d v\left(q^{\prime}\right)
$$

La fonction $(\partial / \partial u)\left|T D^{-1}(u)\right|$ est majorée, si $|Z| \leq 1$. On en déduit que l'opérateur

$$
X \mapsto \int_{M} \nabla_{\mathscr{L} Z}^{\prime}\{K(p, t ; q, 0)[X(q, 0)]\} d v(q)
$$

est majoré uniformément.

L'opérateur $L_{t}^{\prime}$ est la somme des opérateurs définis par les formules (3.10), (3.11), (3.13) et (3.14).

\section{Convergence à l'infini des solutions de l'équation parabolique}

A. Généralisons tout d'abord une remarque de [1, paragraphe 9, B]. Considérons l'opérateur différentiel $\Lambda=\Delta+\partial / \partial t$ sur $C^{\infty}(M \times[0, T[)$.

(4.1) Lemme. Soit $\varphi \in C^{\infty}(M \times[0, T[)$. On suppose $\varphi \geq 0$ et l'existence d'une constante $C$ telle que

$$
\Lambda \varphi(p, t) \leq C \varphi(p, t), \quad \forall(p, t) \in M \times[0, T[.
$$

Soit $\beta, 0<\beta \leq T$. Soit $\nu$ un entier, $\nu>\frac{1}{2} n$.

Il existe alors une constante $K$ et un polynôme $P_{1}$ de degré $\nu-1$ tels que, pour tout $t \geq \beta$, on ait

$$
\varphi_{t} \leq K C^{\nu} \int_{t-\beta}^{t} \int_{M} \varphi(p, u) d v(p) d u+P_{1}(C) \int_{M} \varphi_{t-\beta}(p) d v(p) .
$$

$K$ et $P$ ne dépendent que de $(M, g)$. 
Démonstration. Supposons $\beta \leq T$, sinon il n'y a rien à démontrer. Utilisons la formule de Green associée à $\Lambda$. On a

$$
\left.\varphi_{u}=H_{t-\beta}^{u} * \Lambda \varphi+H_{t-\beta}^{u} \cdot \varphi_{t-\beta}, \quad \forall u \in\right] t-\beta, T[.
$$

De (4.2) et de la positivité du noyau $H$, on déduit

$$
\left.\varphi_{u} \leq C H_{t-\beta}^{u} * \varphi+H_{t-\beta}^{u} \cdot \varphi_{t-\beta}, \quad \forall u \in\right] t-\beta, T[.
$$

et, par récurrence,

$$
\varphi_{t} \leq C^{\nu} H_{t-\beta}^{(\nu) t} * \varphi+\sum_{i=1}^{\nu} C^{i-1} H_{t-\beta}^{(i) t} \cdot \varphi_{t-\beta} .
$$

Dans cette expression, $H^{(i)}$ est l'itérée du noyau $H$ (paragraphe 2, C). Des majorations sur $H^{(i)}$ [9], on déduit que, pour $i>\frac{1}{2} n, H^{(i)}$ est borné sur [0, $T$. Posons

$$
K=\operatorname{Sup} H^{(\nu)}(p, t ; q, 0), \quad p, q \in M, t \in[0, \beta]
$$

et, pour $i, 0<i \leq \nu-1$,

$$
K_{i}=\operatorname{Sup} H^{(i)}(p, \beta ; q, 0), \quad p, q \in M .
$$

$\varphi$ étant supposé positif, on déduit de (4.3)

$$
\varphi_{t} \leq K C^{\nu} \int_{t-\beta}^{t} \int_{M} \varphi+\sum_{i=1}^{\nu} K_{i} C^{i-1} \int_{M} \varphi_{t-\beta} .
$$

(4.4) Remarque. Il est possible de comparer les constantes $K$ et $K_{i}$ à la première valeur propre du laplacien de $(M, g)$.

(4.5) Corollaire. Dans les mêmes conditions que le lemme (4.1), il existe un polynôme $P_{2}$ tel que

$$
\varphi_{t} \leq P_{2}(C) \operatorname{Sup}_{t-\beta \leq u \leq t} \int_{M} \varphi_{u}(p) d v(p) .
$$

B. Dans tout ce paragraphe, on considère une solution $\mu \in C^{\infty}(M \times[0, \infty)$, $\left.M^{\prime}\right)$ du problème (2.1).

(4.6) Lemme. Supposons qu'il existe un réel $\beta_{1}>0$ et un suite $\left\{t_{i} \mid i \in N\right\}$ non-bornée de réels positifs pour lesquels $\mu$ vérifie

i) $\left\{\mu_{t} \mid t \in \bigcup_{i}\left[t_{i}, t_{i}+\beta_{1}\right]\right\}$ est $C^{1}$-borné ;

ii) $\mu_{t_{i+\beta_{1}}} C^{2}$-converge vers une application $\mu_{\infty}$. Alors $\tau\left(\mu_{\infty}\right)=0$.

Démonstration. Sur l'ensemble $\bigcup_{i}\left[t_{i}, t_{i}+\beta_{1}\right]$ la courbure et la densité d'énergie sont bornés. Dans ce cas (voir la formule (5.4)), la fonction $|\partial \mu / \partial t|^{2}$ vérifie une inéquation parabolique. 
On a d'autre part

$$
\int_{M}\left|\dot{\mu}_{t}\right|^{2}=-\frac{d}{d t} E\left(\mu_{t}\right) \geq 0
$$

en notant $\dot{\mu}=\partial \mu / \partial t$. Par conséquent

$$
\sum_{i=1}^{\infty} \int_{t_{i}}^{t_{i}+\frac{1}{2} \beta_{1}} \int_{M}|\dot{\mu}|^{2}=\sum_{i=1}^{\infty}\left[E\left(\mu_{t_{i}}\right)-E\left(\mu_{t_{i}}+\frac{1}{2} \beta_{1}\right)\right]<\infty
$$

On a donc

$$
\lim _{i \rightarrow \infty} \int_{t_{i}}^{t_{i}+\frac{1}{2} \beta_{1}} \int_{M}|\dot{\mu}|^{2}=0
$$

et on peut par conséquent trouver $\beta_{2} \in\left[0, \frac{1}{2} \beta_{1}\right]$ et une sous-suite $j: N \rightarrow N$ tels que

$$
\lim _{i \rightarrow \infty} \int_{M}\left|\dot{\mu}_{t_{j(i)+\beta_{2}}}\right|^{2}=0
$$

Appliquons maintenant le lemme (4.1). On a

$$
\left|\dot{\mu}_{t_{j(i)+\beta_{1}}}\right|^{2} \leq C\left[\int_{t_{j(i)+\beta_{2}}}^{t_{j(i)+\beta_{1}}} \int_{M}|\dot{\mu}|^{2}+\int_{M}\left|\dot{\mu}_{t_{j(i)}+\beta_{2}}\right|^{2}\right]
$$

Ceci implique $C^{0}-\lim _{i \rightarrow \infty} \dot{\mu}_{t_{j(i)+\beta_{1}}}=0$ et donc la conclusion par suite de l'équation de $\mu$.

(4.9) Théorème. Supposons qu'il existe une suite $\left\{t_{i} \mid i \in N\right\}$ non-bornée de réels positifs telle que $\left\{\mu_{t_{i}} \mid i \in N\right\}$ soit $C^{1}$-borné. Alors il existe $\beta$ et une soussuite $j: N \rightarrow N$ telle que $\left\{\mu_{t_{j(i)+\beta}}\right\} C^{k}$-converge vers une application $\mu_{\infty}$. $\mu_{\infty}$ vérifie en outre les propriétés

a) $\mu_{\infty} \in C^{\infty}\left(M, M^{\prime}\right)$,

b) $\tau\left(\mu_{\infty}\right)=0$,

c) $E\left(\mu_{\infty}\right)=\lim _{t \rightarrow \infty} E\left(\mu_{t}\right)$.

Démonstration. Prenons $K=\left\{\mu_{t_{i}} \mid i \in N\right\}$. Du théorème (3.1) on déduit

i) l'existence de $\beta>0$,

ii) $\left\{\mu_{t} \mid t \in \bigcup_{i}\left[t_{i}, t_{i}+\beta\right]\right\}$ est $C^{1}$-borné,

iii) $\left\{\mu_{t_{i}+\beta} \mid i \in N\right\}$ est $C^{k+1}$-borné. Il existe donc une sous-suite convergente.

$\mathrm{Du}$ point iii) on déduit a); au vu de ii) et iii) on peut on peut appliquer le lemme (4.6). Ce qui donne b). Enfin c) est une conséquence immédiate de la. $C^{1}$-convergence.

(4.10) Corollaire. Soit $\mathscr{C}$ une classe d'homotopie d'applications de $M$ dans $M^{\prime}$. Supposons qu'il existe $f \in \mathscr{C}$ telle que 
i) le problème (2.1) admette une solution $\mu \in \mathscr{S}_{\infty}(f)$,

ii) il existe une suite $\left\{t_{i} \mid i \in N\right\}$ non-bornée de réels positifs telle que $\left\{\mu_{t_{i}} \mid i \in N\right\}$ soit $C^{1}$-bornée.

Alors $\mathscr{C}$ contient une application harmonique.

\section{Les théorèmes d'existence et de trivialité des applications harmoniques}

Nous supposerons dans tout ce paragraphe que la courbure sectionnelle de $\left(M^{\prime}, g^{\prime}\right)$ est majorée par un nombre $R>0$. $M$ étant compacte, il existe toujours un réel $r$ tel que l'opérateur de Ricci $S$ de $(M, g)$ vérifie

$$
g(S X, X) \geq \operatorname{rg}(X, X)
$$

Notre définition de ces courbures est telle que pour la sphère $\left(S^{n}, g_{0}\right)$ de rayon 1, la courbure sectionnelle est 1 et l'opérateur de Ricci $(n-1)$ Id. on a dans ce cas particulier $r=n-1$.

De l'équation (2.3) pour $k=1$, on déduit [5] une inéquation vérifiée par l'énergie d'une solution de (2.1):

$$
\Lambda e(\mu) \leq 2\left[R(e(\mu))^{2}-r e(\mu)\right]
$$

(5.3) Remarque. Supposons l'opérateur de Ricci de type positif et. même coercif : on peut choisir $r>0$. Soit $f \in C^{1}\left(M, M^{\prime}\right)$ vérifiant $e(f)<r / R$. Soit $\varepsilon$ tel que $e(f)<r / R-\varepsilon$. Notons [0, $T$ [ l'intervalle maximal tel que $e\left(\mu_{t}\right)<r / R$ $-\varepsilon$. Sur cet intervalle, on a l'inéquation

$$
\Lambda e(\mu) \leq-\varepsilon e(\mu)
$$

et $e\left(\mu_{t}\right)$ est donc majoré par $[r / R-\varepsilon] \exp (-\varepsilon T)$. On a donc $T=\infty$. On a par conséquent dans ce cas

a) la solution $\mu$ de (2.1) est dans $\mathscr{S}_{\infty}(f)$;

b) si $\operatorname{Im}(\mu)$ est bornée dans $\left(M^{\prime}, g^{\prime}\right)$, toute suite $\left\{\mu_{t_{i}} \mid i \in N\right\}$ converge vers une application constante.

Nous utiliserons dans la suite l'inéquation suivante: soit $\gamma$ un majorant de $e(\mu)$. De la démonstration du lemme fondamental de [3], on déduit

$$
\Lambda|\dot{\mu}|^{2} \leq 2 R \gamma|\dot{\mu}|^{2} .
$$

Cette équation a déjà été utilisée au paragraphe 4.

Nous allons donner une généralisation de la remarque (5.3) dans le cas où l'opérateur de Ricci est quelconque. Nous supposerons dans la suite le réel $r \leq 0$. Considérons une solution $\mu \in \mathscr{S}_{\mathrm{T}}(f)$ de $(2.1)$.

(5.5) Lemme. Soit $\gamma_{0}$ un majorant de $e\left(\mu_{t}\right), t \in[0, \beta]$. On peut toujours trouver un réel $\varepsilon(\beta)>0$ tel que si la condition 


$$
R E\left(\mu_{0}\right) \leq \varepsilon(\beta)
$$

est vérifiée, alors il existe une constante r ne dépendant pas de T telle que

$$
e\left(\mu_{t}\right) \leq \gamma, \quad \forall t \in[0, T] .
$$

Soit un réel $E_{0}>0$. On suppose $R \neq 0$.

(5.6) Sous-lemme. $\forall \alpha, 0<\alpha<1, \forall \gamma_{0}>0$, on peut trouver un réel $\varepsilon$ tel que si $E_{0} R \leq \varepsilon$, il existe un réel $\gamma$ vérifiant les deux conditions

i) $\gamma \geq \gamma_{0}$,

ii) $E_{0} P_{2}(2(R \gamma-r)) \leq \alpha \gamma$.

Démonstration. posons $u=R \gamma-r$ et $\lambda=\alpha /\left(R E_{0}\right)$. Les conditions ii) et $\gamma \geq 0$ sont équivalentes à

$$
P_{2}(2 u) \leq \lambda(u+r), \quad u \geq-r .
$$

De même i) est équivalent à

$$
u \geq R \gamma_{0}-r
$$

$P_{2}$ est un polynôme à coefficients positifs, donc convexe. Il possède donc les propriétés suivantes:

i) (5.7) est vérifié pour $u$ pris dans un certain intervalle $\left[u_{1}(\lambda), u_{2}(\lambda)\right]$, $u_{1}(\lambda) \leq u_{2}(\lambda)$; pour $\lambda$ assez grand, cet intervalle n'est pas vide;

ii) si $\lambda^{\prime} \geq \lambda$, on a $\left[u_{1}\left(\lambda^{\prime}\right), u_{2}\left(\lambda^{\prime}\right)\right] \supset\left[u_{1}(\lambda), u_{2}(\lambda)\right]$;

iii) si $\left.\left.\lambda \rightarrow \infty,\left[u_{1}(\lambda), u_{2}(\lambda)\right] \rightarrow\right]-r, \infty\right)$.

Pour $\lambda_{0}$ assez grand, il est donc clair que $R \gamma_{0}-r \leq u_{2}\left(\lambda_{0}\right)$. On pose par conséquent $\varepsilon=\lambda_{0} / \alpha$ et $\gamma \in\left[R \gamma_{0}-r, u_{2}\left(\lambda_{0}\right)\right]$.

Démonstration du lemme (5.5). Soit $\gamma$ comme dans le lemme (5.6). Notons $\left[0, T_{0}\right] \subset[0, T]$ l'intervalle maximal tel que

$$
e\left(\mu_{t}\right) \leq \gamma
$$

On a évidemment $T_{0} \geq \beta$. Appliquons le corollaire (4.5). En posant $C=R \gamma$ $-r, E\left(\mu_{t}\right)$ étant décroissant, il vient

$$
e\left(\mu_{T}\right) \leq E_{0} P_{2}[2(R \gamma-r)] .
$$

D'après le sous-lemme (5.6), on a donc $e\left(\mu_{T}\right) \leq \alpha \gamma$ et $T_{0}$ ne saurait être maximal relativement à la propriété (5.8), si $T_{0} \neq T$. On a donc $T_{0}=T$. Le procédé de calcul montre que $\gamma$ et $\varepsilon(\beta)$ ne dépendent que de $(M, g)$ et pas de $T$. q.e.d.

Le théorème (3.1) s'étend, dans le cas où nous supposons la courbure sectionnelle de $\left(M^{\prime}, g^{\prime}\right)$ majorée, à tout intervalle sur lequel la densité d'énergie est majorée à priori:

(5.9) Lemme. Supposons qu'il existe une constante $C$ telle que si la solu- 
tion $\mu$ de (2.1) existe sur $\left[0, T\left[, T<\infty\right.\right.$, on ait $e\left(\mu_{t}\right) \leq C, \forall t \in[0, T[$. Alors la solution de (2.1) existe sur $[0, T]$.

Démonstration. Du théorème (3.1) et de l'unicité des solutions de (2.1), on déduit de l'existence d'un intervalle maximal $\left[0, T_{0}[\subset[0, T[\right.$ d'existence de la solution.

L'ensemble $\left\{e\left(\mu_{t}\right) \mid t \in\left[0, T_{0}[\}\right.\right.$ est borné. Par application du principe du maximum à l'équation (5.4) [5], on déduit que $|\dot{\mu}|$ est borné et donc que l'ensemble $\operatorname{Im}(\mu)$ est borné dans $\left(M^{\prime}, g^{\prime}\right)$. L'ensemble $\left\{\mu_{t} \mid t \in\left[0, T_{0}[\}\right.\right.$ est donc $C^{1}$-borné. Soit $\alpha$ le réel associé à cet ensemble par le théorème (3.1) (on peut toujours supposer $\alpha \leq T_{0}$ ).

On considère une suite $\left\{t_{i} \mid i \in N\right\}, t_{i} \in\left[0, T_{0}-\alpha\left[, \lim _{i \rightarrow \infty} t_{i}=T_{0}-\alpha\right.\right.$. Une sous-suite $C^{k}$-converge et définit une application $\mu_{T_{0}}$. Mais $|\dot{\mu}|$ étant borné et $T_{0}<\infty$, on a $\mu_{T_{0}}=\lim _{t \rightarrow T_{0}} \mu_{t} \cdot\left[0, T_{0}[\right.$ ne pouvait être maximal, à moins que $T_{0}=T$.

(5.10) Théorème. On peut trouver un réel $e_{1}>0$ tel que pour tout $f \in C^{1}\left(M, M^{\prime}\right)$ vérifiant

$$
\operatorname{Re}(f) \leq e_{1}
$$

on ait:

a) le problème (2.1) admet une solution $\mu$ dans $\mathscr{S}_{\infty}(f)$;

b) si $\operatorname{Im}(\mu)$ est bornée dans $\left(M^{\prime}, g^{\prime}\right)$, pour tout $k \geq 1$ il existe une suite $\left\{t_{i} \mid i \in N\right\}$ nonbornée de réels positifs telle que $\left\{\mu_{t_{i}} \mid i \in N\right\} C^{k}$-converge vers une application harmonique.

Démonstration. Notons $V$ le volume de $(M, g)$. On peut trouver $\varepsilon>0$ tel que si $\operatorname{Re}(f) \leq \varepsilon$, alors la solution de (2.1) existe sur l'intervalle [0,1] ([5] et le lemme (5.9)). D'où une borne supérieure $\gamma_{0}$ pour $e\left(\mu_{t}\right), t \in[0,1]$. Prenons $\left.e_{1}=\operatorname{Inf}\{\varepsilon, \varepsilon(1) / V)\right\}, \varepsilon(1)$ déterminé par le lemme (5.5). $e\left(\mu_{t}\right)$ est donc majoré à priori par $\gamma$ et du lemme (5.9), on déduit l'existence de la solution sur tout intervalle $[0, T[$. Le point $b$ ) est une conséquence immédiate du théorème (4.9). q.e.d.

Supposons pour terminer que la variété $(M, g)$ posséde la propriété suivante

$$
V P_{2}(-r)<1 \text {. }
$$

$P_{2}$ étant le polynôme du lemme (4.5).

(5.12) Théorème. Si $(M, g)$ vérifie la condition (5.11), on peut trouver un réel $e_{2}>0$ tel que si la condition

$$
\operatorname{Re}(f) \leq e_{2}
$$

est vérifiée, alors les conclusions du théorème (5.10) sont vraies et $\mu_{\infty}$ est une application constante.

Démonstration. on a $E\left(\mu_{t}\right) \leq V\left\|e\left(\mu_{t}\right)\right\|_{L^{\infty}(M)}$. Du lemme (4.5) on déduit 
$e\left(\mu_{t+1}\right) \leq V e\left(\mu_{t}\right) P_{2}[2(R \gamma-r)]$. Considérons un réel $\varepsilon>0$ tel que $V P_{2}(-r)<$ $1-\varepsilon$. Appliquons le principe du maximum à l'équation (5.2) [5]. On peut trouver $\varepsilon^{\prime}$ tel que si $\operatorname{Re}(f) \leq \varepsilon^{\prime}$, alors $e\left(\mu_{t+u}\right) \leq(1+\varepsilon) e\left(\mu_{t}\right), \forall u \in[t, t+1]$.

Pour tout $\gamma \leq \gamma_{0}, \gamma_{0}$ assez petit: $R \gamma_{0} \leq \varepsilon^{\prime \prime}$, on a $V P_{2}[2(R \gamma-r)] \leq 1-\varepsilon$. On en déduit

$$
e\left(\mu_{t+1}\right) \leq(1-\varepsilon) e\left(\mu_{t}\right),
$$

pourvu que $R(1+\varepsilon) e\left(\mu_{t}\right) \leq \varepsilon^{\prime \prime}$. Posons $e_{2}=\operatorname{Inf}\left\{\varepsilon^{\prime}, \varepsilon^{\prime \prime} /(1+\varepsilon)\right\}$. On déduit de (5.13) que $e\left(\mu_{t}\right)$ est majorée par une fonction décroissante $\varphi$ du paramètre, $\lim _{t \rightarrow \infty} \varphi(t)=0$. On a donc $C^{0}-\lim _{t \rightarrow \infty} e\left(\mu_{t}\right)=0$. q.e.d.

Les théorèmes (1.3) et (1.4) sont des corollaires immédiats des théorèmes (5.10) et (5.12)

\section{Bibliographie}

[1] J. Eells \& J. H. Sampson, Harmonic mappings of Riemannian manifolds, Amer. J. Math. 86 (1964) 109-160.

[ 2 ] H. I. Eliasson, Geometry of manifolds of maps, J. Differential Geometry 1 (1967) 169-194.

[ 3 ] P. Hartman, Homotopic harmonic maps, Canad. J. Math. 19 (1967) 673-687.

[ 4 ] A. Lichnerowicz, Propagateurs et commutateurs en relativite générale, Inst. Hautes Études Sci. Publ. Math. No. 10 (1960).

[ 5 ] J. C. Mitteau, Sur les solutions de l'équation de la chaleur, C. R. Acad. Sci. Paris 270 (1970) 1244-1247.

[6] - L'équation parabolique sur les applications de variétés, Thèse.

[7] _- Sur l'existence des extrémales de l'énergie, C. R. Acad. Sci. Paris 274 (1972) 1499-1501.

[8] R. S. Palais, Foundations of global non-linear analysis, Benjamin, New York, 1968.

[9] W. Pogorzelski, Étude de la solution fondamentale de l'équation parabolique, Ricerca (Napoli) 5 (1956) 25-56.

[10] — Propriétés des intégrales de l'équation parabolique normale, Ann. Polon. Math. 4 (1957) 61-92. 